Research Article

\title{
Forecasting the Number of the Wounded after an Earthquake Disaster Based on the Continuous Interval Grey Discrete Verhulst Model
}

\author{
Jun Zhang $\mathbb{D}^{1},{ }^{1}$ Tongyuan Wang $\mathbb{D}{ }^{2}{ }^{2}$ Jianpeng Chang $\mathbb{D},{ }^{1}$ and Yan Gou ${ }^{3}$ \\ ${ }^{1}$ School of Management Science and Engineering, Chongqing Technology and Business University, Chongqing 400067, China \\ ${ }^{2}$ School of Business, Henan University, Kaifeng 475001, China \\ ${ }^{3}$ School of International Business, Chongqing Technology and Business University, Chongqing 400067, China
}

Correspondence should be addressed to Tongyuan Wang; tongyuan_wang@163.com

Received 19 November 2020; Revised 30 January 2021; Accepted 22 March 2021; Published 5 April 2021

Academic Editor: Wen Yi

Copyright (c) 2021 Jun Zhang et al. This is an open access article distributed under the Creative Commons Attribution License, which permits unrestricted use, distribution, and reproduction in any medium, provided the original work is properly cited.

Earthquake disaster causes serious casualties, so the prediction of casualties is conducive to the reasonable and efficient allocation of emergency relief materials, which plays a significant role in emergency rescue. In this paper, a continuous interval grey discrete Verhulst model based on kernels and measures (CGDVM-KM), different from the previous forecasting methods, can help us to efficiently predict the number of the wounded in a very short time, that is, an "S-shape" curve for the numbers of the sick and wounded. That is, the continuous interval sequence is converted into the kernel and measure sequences with equal information quantity by the interval whitening method, and it is combined with the classical grey discrete Verhulst model, and then the grey discrete Verhulst models of the kernel and measure sequences are presented, respectively. Finally, CGDVM-KM is developed. It can effectively overcome the systematic errors caused by the discrete form equation for parameter estimation and continuous form equation for simulation and prediction in classical grey Verhulst model, so as to improve the prediction accuracy. At the same time, the rationality and validity of the model are verified by examples. A comparison with other forecasting models shows that the model has higher prediction accuracy and better simulation effect in forecasting the wounded in massive earthquake disasters.

\section{Introduction}

In recent years, frequent earthquake disasters have occurred frequently all over the world. Due to the uncertainty and destructiveness of earthquake, it has caused a great loss of people and property. For example, Haiti (7.3 magnitude on January 13th, 2010, killed 222500 people and injured 196000 people), Chile (8.8 magnitude on February 27th, 2010, killed more than 750 people), Japan (8.6 magnitude on March 11th, 2011, killed 19533 people and missed 2585 people), in China, Wenchuan (8.0 magnitude on May 12th, 2008, killed 69227 people, injured 374643 and missed 17923 people), and Yushu (7.1 magnitude on April 14th, 2010, killed 2698 people). These data are the official report of the massive earthquakes from China Seismological Bureau (https:// www.cea.gov.cn/cea/dzpd/index.html). It can be seen that the occurrence of earthquake disasters has exerted a serious impact on people's production and life and also caused heavy casualties and economic losses. However, due to the sudden and fragility of earthquake disaster, it becomes difficult and challenging to prevent in advance. Measures can be taken to effectively deal with the disaster after it occurs, so as to reduce casualties and property losses $[1,2]$.

Massive earthquake disaster has the characteristics of strong suddenness and weak prediction, and its occurrence is often accompanied by road collapse, channel obstruction, and other problems, which cause inconvenience to rescue operations, so that emergency relief materials cannot be delivered timely and fully after the disaster. Therefore, it is necessary to accurately predict the emergency materials (such as medical materials needed to treat the wounded), and the premise of forecasting is to know the number of the wounded every day. 
Through the forecasting of the number of the wounded, we can not only indirectly predict the demand for drugs in earthquake disaster emergency rescue but also play a guiding role in the allocation of relief materials.

Choosing a suitable mathematical model is key to achieving accurate prediction of the number of the wounded. After the occurrence of the massive earthquake disasters, only a small amount of information can be available for reference to the prediction of the number of the wounded in a short time. In view of this kind of information, the grey prediction model can play a very good prediction effect. Grey system prediction method is based on people's understanding of the uncertainty characteristics of system evolution, aiming at the problems of grey uncertainty prediction existing in reality, using a small amount of effective data, mining the inherent evolution law of the system, and then making a scientific prediction of the development trend of the system [3]. Therefore, we build a grey prediction model to forecast the number of the wounded after the massive earthquake disasters.

In this paper, we first analyze the previous data and find that the data of the wounded present the characteristics of "S-shape" and continuous interval dynamic change. According to the characteristics of data, interval grey number whitening method and grey discrete Verhulst method are selected to construct continuous interval grey number discrete Verhulst prediction model based on kernels and measures (CGDVM-KM), which is used to simulate and forecast the number of the wounded in Lushan earthquake of Sichuan Province in China, and good results are obtained. The grey prediction model proposed in this paper can provide a good support for the emergency rescue of massive earthquake disasters, so as to provide reference for the later material allocation of emergency rescue.

The main contributions of this paper are as follows. First, we use interval bleaching principle of converting continuous interval sequence information, such as nuclear and measure sequences, and then, it is combined with grey Verhulst model, and a nuclear sequence grey Verhulst model and measure is established. A continuous discrete Verhulst grey forecasting model is rebuilt which can effectively overcome errors done by the classical grey Verhulst model due to the parameter estimation using discrete simulation and prediction equation using continuous form equation of system error and improve the prediction precision. Second, due to the suddenness and persistence of major earthquakes, the number of diseases and injuries shows certain grey characteristics, such as the presence of known or unknown information, and the sample size is small and limited. Therefore, we will construct the CGDVM-KM to predict the number of illnesses and injuries in the early stage of an earthquake, as well as the need for emergency medicines. Through comparative analyses with several other grey prediction models, it is found that the model constructed in this paper can well fit the number of the wounded in the massive earthquake disasters, so as to accurately forecast the number of the wounded in a period of time in the future, and provide reference for emergency rescue and later material allocation.
The rest of this paper is organized as follows. Section 2 reviews the related literature. The data characteristics and methods used are introduced in Section 3. Section 4 presents the required forecast model. Section 5 forecasts the number of the wounded and makes comparative analysis. The conclusions are drawn in Section 6.

\section{Literature Review}

There are two aspects of literature related to this study: (1) the forecasting of the wounded in the massive earthquake disasters; (2) grey prediction model.

\subsection{Forecasting the Wounded in Massive Earthquake} Disasters. In the literature of the forecasting of the number of the wounded in the earthquake disasters, Wu and Gu used the modified exponential curve to fit the data and predict the number of death in Wenchuan earthquake in China [4]. Wang et al. established a BP neural network model to predict casualties in earthquakes. The model examined the key factors, such as earthquake magnitude, focal depth, epicenter intensity, disaster preparedness level, earthquake acceleration, population density, and disaster prediction, and used 37 severe earthquake disasters to train the network. The results show that the model is applicable to most earthquake situations [5]. Zhang et al. proposed to use the grey discrete Verhulst model to predict the drug supplies for emergency rescue of large-scale earthquake disasters [2]. Gul and Guneri established an artificial neural network (ANN) model for earthquake casualty prediction, which takes the time of earthquake occurrence, earthquake magnitude, and population density as the prediction factors, and use five earthquake disasters occurring in Turkey since 1975 as the network training sample [6]. Huang et al. introduced extreme learning machine (ELM) into earthquake casualty prediction. Through data training, the ELM network structure for earthquake casualty prediction was established, and the number of hidden layer nodes and excitation function was determined to ensure the reliability of ELM network prediction results [7]. Furthermore, Huang et al. proposed an adaptive chaotic particle swarm optimization (ACPSO) to optimize network parameters of traditional ELM to improve the stability and prediction accuracy of the network and apply the improved elm model to the prediction of casualties in earthquake disasters [8]. Firuzi et al. proposed an empirical model for mortality estimation in Iran based on vibration related parameters (PGA) [9].

As we all know, the neural network and extreme learning machine prediction methods need more original data for training and learning in advance. However, the emergency rescue of earthquake disaster is more urgent, requiring longer time and smaller sample size, so it is not applicable. However, the grey system model used in literature [2] only uses grey Verhulst model to predict the number of the wounded in real number, which does not fully reflect the characteristics of continuous interval of the number of patients. This paper not only takes into account the saturated " $S$ " type change trend of the number of wounded and sick 
people but also fully considers the characteristics of continuous interval of the number of wounded and sick people and uses the improved interval grey Verhulst model to predict the number of the wounded after the occurrence of earthquake disasters, so that the prediction accuracy can be improved.

2.2. Grey Prediction Models. Many scholars have studied the grey Verhulst prediction model. Zhang et al. introduced the grey Verhulst model into the field of load forecasting and the application of grey Verhulst model in medium and longterm load forecasting through typical examples [10]. Wang et al. proposed an unbiased grey Verhulst model for the inherent simulation error of grey Verhulst model [11]. Cui et al. proposed the grey discrete Verhulst model according to the incomplete adaptability of the traditional grey Verhulst model, referring to the idea of discretization, and through mutual generation of the original data [12]. Hashem-Nazari et al. proposed the direct basic form-focused nonequidistant grey Verhulst model and effectively predicted the socioconomic time series focusing on the population of Iran [13]. Wang and Li built a derived non-equigap grey Verhulst model and explore the relationship between carbon dioxide emissions and economic growth. Empirical research shows that the relationship between carbon dioxide emissions and economic growth has an inverted U-shaped curve [14]. Rajesh used the grey Verhulst model to determine the driving factors of social and environmental risk management (SERM) in an elastic supply chain, so as to effectively improve the performance of enterprise SERM [15]. Wu and $\mathrm{Xu}$ used the grey Verhulst model to predict the comprehensive air quality index [16]. Zeng et al. used the improved grey Verhulst model to reasonably predict tight gas production [17]. Tian et al. used the Verhulst grey model to predict the value of ocean carrying capacity in the next five years (2017-2021) [18]. Zhao et al. predicted the number of patients with COVID-19 infection in China based on grey Verhulst model [19].

The traditional discrete GM $(1,1)$ model does not always fit well, and sometimes the prediction error is large $[20,21]$, so some scholars began to study interval grey numbers. Zeng et al. developed a DGM $(1,1)$ prediction model for interval grey number series [22]. According to the geometric characteristics of interval grey number sequence on two-dimensional coordinate plane, Zeng and Liu proposed a prediction model of interval grey number based on its geometrical characteristics through area transformation and coordinated transformation to transform interval grey number sequence into real number sequence without information loss. Thus, the interval grey number sequence with uncertain information is simulated and predicted [23]. Considering that some data are fuzzy or missing after earthquake disasters, which leads to difficulties in material demand prediction, Zhang et al. proposed using the fuzzy interval grey number for prediction to improve the prediction accuracy [24]. Li et al. proposed a new operation rule of grey interval number multiplication, which improved the accuracy of grey number division. Then, on the basis of the proposed calculation rules, combined with grey reduction preprocessing, the traditional grey heterogeneous data prediction modeling method is improved and applied to the inventory replenishment scheduling problem in emergency rescue scenarios [25]. Zeng et al. established a new interval grey number prediction model through the kernel function of grey number band and regional sequence [26]. Zeng et al. divided the interval grey numbers into two real number parts, namely, the "white" and "grey" parts. Then, grey Verhulst model and DGM $(1,1)$ model are developed to simulate and predict the "white" and "grey" parts, respectively. Thus, the problem of expanding the range of interval grey number is solved to a certain extent [27]. Furthermore, according to the situation that the traditional grey correlation model cannot extract interval feature information from the interval grey number sequence, Ye et al. built a grey correlation analysis model (GRA) based on effective information transformation of interval grey number. Then, a multivariate GM $(1, n)$ model is developed to predict the interval grey number series [28].

The aforementioned literature has optimized and applied the grey Verhulst model and interval grey number prediction model from various perspectives, but the scope of application of each model is not the same. None of the aforementioned models is appropriate for predicting the number of the wounded in the massive earthquake disasters. Considering that the number of wounded people after an earthquake is changing in a continuous interval, this paper will use the interval bleaching principle of converting continuous interval sequence information, such as nuclear sequence, and the measure which build nuclear sequence grey Verhulst model and measure, the final reduction build a continuous discrete Verhulst grey forecasting model. After the subsequent empirical test, the improved model is more effective and accurate in predicting the number of earthquake wounded.

\section{Problem Descriptions}

In this section, we analyzed the data of the number of the wounded after the massive earthquake disasters and then built a corresponding model to forecast according to the data characteristics.

3.1. Data Characteristics. In order to find the statistical law of the number of the wounded in the massive earthquake disasters, the statistical data of early earthquake disasters in Yushu, Qinghai Province, and Lushan, Sichuan Province, in China are selected. The statistical data curve is drawn and shown in Figure 1. These data are semidaily data, based on the official report of these two massive earthquakes from China Seismological Bureau (https://www.cea.gov.cn/cea/ dzpd/index.html).

Through the statistical data analysis of the number of patients after the massive earthquake disaster, it can be concluded that there are two characteristics in the statistical data of the number of the wounded. 




Figure 1: The number of the wounded in the early stage of earthquake.

3.1.1. Saturated "S-Shape" Characteristics. From Figure 1, we can see that, at the beginning of the rescue, the number of the wounded shows an exponential rapid growth (i.e., rapid growth period). With further development of the rescue operation, the number of newly discovered wounded people gradually decreased, and the growth rate gradually slowed down. Meanwhile, some wounded with minor injuries gradually recovered and withdrew from treatment, and some patients who were seriously injured would be sent to large hospitals to receive further treatment after simple treatment, so the number of the wounded gradually stabilized at a total amount and no longer increased (i.e., stationary period). As a whole, it shows a saturated "S-shape" change trend. At the same time, the real-time statistical data of the number of the wounded has been carried out at the beginning of the rescue operation, and it is easy to get along with the rescue operation.

3.1.2. Continuous Interval Dynamic Variability. The statistics of the number of the wounded can be divided into point data and interval data. The point data only reflects the number of the wounded found at the end of the recording time point of the day. In the golden rescue period, the search and rescue process is 24-hour without interruption. During and after the statistical process, wounded people were continuously found. The number is increasing every hour, which is a dynamic and continuous growth and changing process, and the number of the wounded has a continuous interval every hour and every day. Therefore, point data cannot accurately describe the real situation. The data of the wounded can be regarded as a continuous change interval, which can reflect the reality more truly and accurately.

3.2. Method. The construction of continuous interval grey number prediction model involves two problems. One is the whitening treatment of continuous interval grey number. That is, the continuous interval grey number sequence is transformed into real number sequence with equal amount of information. The other is to select the model aiming at the data characteristics of the real number. In the kernel and measure method, the midpoint of the lower bound of the interval is selected as the kernel sequence, and the interval length is selected as the measure sequence. The interval grey number is transformed into two real number sequences of kernels and measures, which can avoid the problems caused by the direct operation of interval grey number and make full use of all the information contained in the interval grey number. Therefore, it can well predict the interval grey number.

Verhulst model describes a dynamic development process, which initially shows an exponential rapid growth. As time goes by, the growth rate gradually slows down and finally decreases to zero due to the interference of some external factors, and the total amount is stable at a fixed value. That means the model is mainly used to describe the grey dynamic change process of the whole data with saturated "S-shape" feature. From the modeling process of the classical grey Verhulst model, we can see that there are some errors in the conversion process from the whitening equation to the final response formula, and the grey discrete Verhulst model can effectively reduce this error. Consequently, in view of the characteristics of saturated "S-shape" and continuous change interval in the number of the wounded in the massive earthquake disaster, this paper analyses the selection of prediction methods and interval whitening method and proposes the continuous interval grey discrete Verhulst model based on kernels and measures (CGDVM-KM) to simulate and predict the number of the wounded in Lushan earthquake, Sichuan Province, in China.

\section{Construction of the Prediction Model}

The basic idea of constructing continuous interval grey discrete Verhulst model based on kernels and measures is as follows. First, we whiten all the grey elements of continuous interval grey number sequence and then transform them into equal information of kernel sequence and measure sequence. Second, grey discrete Verhulst model of kernel and measure sequences is constructed to predict the kernels 
and measures. Finally, we deduced and restored the upper and lower bounds of the continuous interval grey number, and then, the prediction of the continuous interval grey number is realized.

For the continuous interval grey number, the kernel is the center of the interval, reflecting the development trend of the continuous interval grey number sequence, which is expressed as half of the sum of the upper and lower bound interval data. The measure is the length of the continuous interval, reflecting the information mastering the continuous interval grey number sequence, and the numerical value is the difference between the upper and lower bounds. According to the literature of $[12,29]$, we have the following definitions.

Definition 1. Suppose a continuous interval $\left[a_{k}, b_{k}\right]$, and grey number $\otimes \in\left[a_{k}, b_{k}\right]$. We considered that $\widetilde{\otimes}_{k}=\left(a_{k}+\right.$ $\left.b_{k}\right) / 2$ is the kernel of the continuous interval, and $l\left(\otimes_{k}\right)=$ $b_{k}-a_{k}$ is the measure of the continuous interval.

Definition 2. Suppose the original sequence as $X^{(0)}=$ $\left(x^{(0)}(1), x^{(0)}(2), \ldots, x^{(0)}(k)\right)$. Here, $X^{(0)}(k)$ indicates the data at time $k$. Suppose that $Y^{(0)}=\left(y^{(0)}(1)\right.$, $\left.y^{(0)}(2), \ldots, y^{(0)}(n)\right)$ is the reciprocal of $X^{(1)}$, where $X^{(1)}$ is the first aggregate generator operator $(1-\mathrm{AGO})$ of $X^{(0)}$. Then, the following is obtained: $y^{(0)}(k)=1 / x^{(1)}(k)(k=$ $1,2, \ldots, n)$. Suppose that $Y^{(1)}=\left(y^{(1)}(1), y^{(1)}(2), \ldots\right.$, $\left.y^{(1)}(n)\right)$ is the 1-AGO of $Y^{(0)}$, and $y^{(1)}(k)=\sum_{i=1}^{k} y^{(0)}(i)$. Thus, the grey discrete Verhulst model is

$$
y^{(1)}(k+1)=\beta_{1}+\beta_{2} k+\beta_{3} y^{(1)}(k) \text {. }
$$

We can obtain the estimated parameters $\beta=\left(\beta_{1}, \beta_{2}, \beta_{3}\right)^{T}$ as follows:

$$
\left\{\begin{array}{l}
\beta_{1}=\frac{\sum_{k=1}^{n-1} y^{(1)}(k+1)}{n-1}-\frac{n}{2} \beta_{2}-\frac{\sum_{k=1}^{n-1} y^{(1)}(k)}{n-1} \beta_{3}, \\
\beta_{2}=\frac{6 n \sum_{k=1}^{n-1} y^{(1)}(k+1)-\sum_{k=1}^{n-1}\left(k \cdot y^{(1)}(k+1)\right)}{n(n-1)(n-2)}-\frac{3 n \sum_{k=1}^{n-1} y^{(1)}(k)-6 \sum_{k=1}^{n-1}\left(k \cdot y^{(1)}(k)\right)}{3 n \sum_{k=1}^{n-1} y^{(1)}(k)-n(n-1)(2 n-1)} \beta_{3}, \\
\beta_{3}=\frac{n(n-1)(n-2) \sum_{k=1}^{n-1}\left(y^{(1)}(k+1) \cdot y^{(1)}(k)\right)}{12 n \sum_{k=1}^{n-1} y^{(1)}(k) \cdot \sum_{k=1}^{n-1}\left(k \cdot y^{(1)}(k)\right)+n(n-1)(n-2) \sum_{k=1}^{n-1}\left(y^{(1)}(k)\right)^{2}-2 n(2 n-1)\left(\sum_{k=1}^{n-1} y^{(1)}(k)\right)^{2}-12\left(\sum_{k=1}^{n-1} k \cdot y^{(1)}(k)\right)^{2}} \\
+\frac{12 n \sum_{k=1}^{n-1}\left(y^{(1)}(k)\right) \cdot \sum_{k=1}^{n-1}\left(k \cdot y^{(1)}(k)\right)+n(n-1)(n-2) \sum_{k=1}^{n-1}\left(y^{(1)}(k)\right)^{2}-2 n(2 n-1) \sum_{k=1}^{n-1}\left(y^{(1)}(k)\right)^{2}-12\left(\sum_{k=1}^{n-1} k \cdot y^{(1)}(k)\right)^{2}}{\cdot 2 n(2 n-1) \sum_{k=1}^{n-1}\left(y^{(1)}(k)\right) \cdot \sum_{k=1}^{n-1}\left(y^{(1)}(k+1)\right)} \\
\cdot 12 n \sum_{k=1}^{n-1}\left(y^{(1)}(k)\right) \cdot \sum_{k=1}^{n-1}\left(k \cdot y^{(1)}(k)\right)+n(n-1)(n-2) \sum_{k=1}^{n-1}\left(y^{(1)}(k)\right)^{2}-2 n(2 n-1) \sum_{k=1}^{n-1}\left(y^{(1)}(k)\right)^{2}-12\left(\sum_{k=1}^{n-1} k \cdot y^{(1)}(k)\right)^{2} \\
-\frac{12\left(\sum_{k=1}^{n-1} k \cdot y^{(1)}(k)\right) \cdot\left(\sum_{k=1}^{n-1} k \cdot y^{(1)}(k+1)\right)}{12 n \sum_{k=1}^{n-1}\left(y^{(1)}(k)\right) \cdot \sum_{k=1}^{n-1}\left(k \cdot y^{(1)}(k)\right)+n(n-1)(n-2) \sum_{k=1}^{n-1}\left(y^{(1)}(k)\right)^{2}-2 n(2 n-1) \sum_{k=1}^{n-1}\left(y^{(1)}(k)\right)^{2}-12\left(\sum_{k=1}^{n-1} k \cdot y^{(1)}(k)\right)^{2}} .
\end{array}\right.
$$

The time response function of the grey discrete Verhulst model is

$$
\hat{x}^{(1)}(k+1)=\frac{1}{\hat{y}^{(0)}}(k+1)= \begin{cases}{\left[k \beta_{2}+\beta_{1}\right]^{-1},} & \beta_{3}=1, \\ {\left[\beta_{1} \beta_{3}^{k-1}+\frac{\beta_{2}\left(1-\beta_{3}^{k}\right)}{1-\beta_{3}}-\beta_{3}^{k-1}\left(1-\beta_{3}\right) \frac{1}{x^{(0)}(1)}\right]^{-1},} & \beta_{3} \neq 1 .\end{cases}
$$


Finally, the grey discrete Verhulst model based on kernels and measures is constructed as follows.

4.1. Prediction of the Kernels. From Definition 1, we can obtain the original sequence of kernel sequence:

$$
X^{(0)}(\widetilde{\otimes})=\left(\widetilde{\otimes}_{1}, \widetilde{\otimes}_{2}, \ldots, \widetilde{\otimes}_{n}\right) .
$$

The kernel sequence's time response function of the grey discrete Verhulst model is

$$
\begin{aligned}
\widehat{a}(k+1) & =\widehat{\otimes}^{(1)}(k+1) \\
& =\left[\alpha_{1} \alpha_{3}^{k-1}+\frac{\alpha_{2}\left(1-\alpha_{3}^{k}\right)}{1-\alpha_{3}}-\alpha_{3}^{k-1}\left(1-\alpha_{3}\right) \frac{1}{\widetilde{\otimes}^{(0)}(1)}\right]^{-1} .
\end{aligned}
$$

According to (5), the kernel sequence at the corresponding time can be predicted by substituting a different time $k$.

4.2. Prediction of the Measures. From Definition 1, we can obtain the original sequence of measure sequence:

$$
L^{(0)}=\left(l^{(0)}(1), l^{(0)}(2), \ldots, l^{(0)}(k)\right) .
$$

$L^{(0)}(k)$ indicates the number of the wounded at time $k$, and $L^{(1)}$ is the first aggregate generator operator (1-AGO) of $L^{(0)}$.

The measure sequence's time response function of the grey discrete Verhulst model is

$$
\widehat{l}^{(1)}(k+1)=\left[\beta_{1} \beta_{3}^{k-1}+\frac{\beta_{2}\left(1-\beta_{3}^{k}\right)}{1-\beta_{3}}-\beta_{3}^{k-1}\left(1-\beta_{3}\right) \frac{1}{l^{(0)}(1)}\right]^{-1} .
$$

It can be obtained by reduction:

$$
\begin{aligned}
\hat{l}^{(0)}(k+1)= & {\left[\beta_{1} \beta_{3}^{k-1}+\frac{\beta_{2}\left(1-\beta_{3}^{k}\right)}{1-\beta_{3}}-\beta_{3}^{k-1}\left(1-\beta_{3}\right) \frac{1}{l^{(0)}(1)}\right]^{-1} } \\
& -\left[\beta_{1} \beta_{3}^{k-2}+\frac{\beta_{2}\left(1-\beta_{3}^{k-1}\right)}{1-\beta_{3}}-\beta_{3}^{k-2}\left(1-\beta_{3}\right) \frac{1}{l^{(0)}(1)}\right]^{-1} .
\end{aligned}
$$

According to (8), the measure sequence at the corresponding time can be predicted by substituting a different time $k$.

4.3. Prediction Model of Upper and Lower Bounds of Continuous Interval Grey Number. The prediction values of kernels and measures sequences are solved by Definition 2 .

$$
\left\{\begin{array}{l}
\widehat{a}(k+1)+\widehat{b}(k+1)=2 \widehat{\widehat{\otimes}}(k+1), \\
\widehat{b}(k+1)-\widehat{a}(k+1)=\widehat{l}(k+1) .
\end{array}\right.
$$

According to (5), the grey prediction model of upper and lower bounds of interval grey number $\widehat{\otimes}(k+1) \epsilon$ $[\widehat{a}(k), \widehat{b}(k)]$ can be obtained.

$$
\begin{aligned}
& \left\{\begin{array}{l}
\widehat{a}(k+1)=\widehat{\widetilde{\otimes}}(k+1)-\frac{1}{2} \widehat{l}(k+1), \\
\widehat{b}(k+1)=\widehat{\widetilde{\otimes}}(k+1)+\frac{1}{2} \widehat{l}(k+1),
\end{array}\right. \\
& \widehat{a}(k+1)=\left[\alpha_{1} \alpha_{3}^{k-1}+\frac{\alpha_{2}\left(1-\alpha_{3}^{k}\right)}{1-\alpha_{3}}-\alpha_{3}^{k-1}\left(1-\alpha_{3}\right) \frac{1}{\widetilde{\Phi}^{(0)}(1)}\right]^{-1}-\frac{1}{2}\left(\left[\beta_{1} \beta_{3}^{k-1}+\frac{\beta_{2}\left(1-\beta_{3}^{k}\right)}{1-\beta_{3}}-\beta_{3}^{k-1}\left(1-\beta_{3}\right) \frac{1}{l^{(0)}(1)}\right]^{-1}\right. \\
& \left.-\left[\beta_{1} \beta_{3}^{k-2}+\frac{\beta_{2}\left(1-\beta_{3}^{k-1}\right)}{1-\beta_{3}}-\beta_{3}^{k-2}\left(1-\beta_{3}\right) \frac{1}{l^{(0)}(1)}\right]^{-1}\right) \\
& \widehat{b}(k+1)=\left[\alpha_{1} \alpha_{3}^{k-1}+\frac{\alpha_{2}\left(1-\alpha_{3}^{k}\right)}{1-\alpha_{3}}-\alpha_{3}^{k-1}\left(1-\alpha_{3}\right) \frac{1}{\bar{\otimes}^{(0)}(1)}\right]^{-1}-\frac{1}{2}\left(\left[\beta_{1} \beta_{3}^{k-1}+\frac{\beta_{2}\left(1-\beta_{3}^{k}\right)}{1-\beta_{3}}-\beta_{3}^{k-1}\left(1-\beta_{3}\right) \frac{1}{l^{(0)}(1)}\right]^{-1}\right. \\
& \left.-\left[\beta_{1} \beta_{3}^{k-2}+\frac{\beta_{2}\left(1-\beta_{3}^{k-1}\right)}{1-\beta_{3}}-\beta_{3}^{k-2}\left(1-\beta_{3}\right) \frac{1}{l^{(0)}(1)}\right]^{-1}\right) .
\end{aligned}
$$


Equation (11) is the upper and lower bound prediction model of continuous interval grey number. The interval number of the wounded can be predicted by substituting it into different time intervals $k$. Finally, the average relative error and synthetic average relative simulation error can be obtained, respectively.

4.4. Error Test Standard. After using the prediction method to fit the curve, we need to use the relative error index to test the fitting degree.

Definition 3. Suppose that the sequence of the original continuous interval grey numbers is [30]

$$
\begin{aligned}
X^{(0)} & =\left(x^{(0)}(1), x^{(0)}(2), \ldots, x^{(0)}(n)\right) \\
& =\left(\left[a_{1}, b_{1}\right],\left[a_{2}, b_{2}\right], \ldots,\left[a_{n}, b_{n}\right]\right) .
\end{aligned}
$$

The simulated sequence of the model is as follows:

$$
\begin{aligned}
\widehat{X}^{(0)} & =\left(\widehat{x}^{(0)}(1), \widehat{x}^{(0)}(2), \ldots, \widehat{x}^{(0)}(n)\right) \\
& =\left(\left[\widehat{a}_{1}, \widehat{b}_{1}\right],\left[\widehat{a}_{2}, \widehat{b}_{2}\right], \ldots,\left[\widehat{a}_{n}, \widehat{b}_{n}\right]\right) .
\end{aligned}
$$

The lower bound and upper bound residual sequences are

$$
\left\{\begin{array}{l}
\varepsilon_{a}=\left(\varepsilon_{a}(1), \varepsilon_{a}(2), \ldots, \varepsilon_{a}(n)\right)=\left(a_{1}-\widehat{a}_{1}, a_{2}-\widehat{a}_{2}, \ldots, a_{n}-\widehat{a}_{n}\right), \\
\varepsilon_{b}=\left(\varepsilon_{b}(1), \varepsilon_{b}(2), \ldots, \varepsilon_{b}(n)\right)=\left(b_{1}-\widehat{b}_{1}, b_{2}-\widehat{b}_{2}, \ldots, b_{n}-\widehat{b}_{n}\right) .
\end{array}\right.
$$

Finally, we calculate the relative error sequence of the lower and upper bounds:

$$
\left\{\begin{array}{l}
\Delta_{a}=\left(\Delta_{a}(1), \Delta_{a}(2), \ldots, \Delta_{a}(n)\right)=\left(\left|\frac{\varepsilon_{a}(1)}{a_{1}}\right|,\left|\frac{\varepsilon_{a}(2)}{a_{2}}\right|, \ldots,\left|\frac{\varepsilon_{a}(n)}{a_{n}}\right|\right), \\
\Delta_{b}=\left(\Delta_{b}(1), \Delta_{b}(2), \ldots, \Delta_{b}(n)\right)=\left(\left|\frac{\varepsilon_{b}(1)}{b_{1}}\right|,\left|\frac{\varepsilon_{b}(2)}{b_{2}}\right|, \ldots,\left|\frac{\varepsilon_{b}(n)}{b_{n}}\right|\right) .
\end{array}\right.
$$

(1) For $k \leq n$, we considered that $\Delta_{a}(k)=\left|\varepsilon_{a}(k) / a_{k}\right|$ is the simulate the relative error at the point $k$ of the lower bound sequence, and $\bar{\Delta}_{a}=(1 / n) \sum_{k=1}^{n} \Delta_{a}(k)$ is the average relative error of the lower bound sequence. The upper bound error calculation is omitted for similarity.

(2) $\bar{\Delta}=(1 / 2)\left(\bar{\Delta}_{a}+\bar{\Delta}_{b}\right)$ is called the comprehensive average model relative error.

(3) For $\alpha$, when $\bar{\Delta}<\alpha$ and $\Delta_{a}(n)<\alpha, \Delta_{b}(n)<\alpha$ hold, the model is a residual qualified model.

The prediction results must overpass the accuracy test to judge whether the result is qualified and fits its accuracy level. Only the prediction model, which has overpassed the accuracy test, can ensure the rationality and accuracy of its prediction value. The lower the stage is, the better the prediction effect will be. By calculating the residual error and relative error of each point, the average simulation relative
TABLE 1: Continuous interval sequence error test table.

\begin{tabular}{lc}
\hline Accuracy classes & Average relative error $\alpha$ \\
\hline Stage 1 & 0.01 \\
Stage 2 & 0.05 \\
Stage 3 & 0.10 \\
Stage 4 & 0.20 \\
\hline
\end{tabular}

TABLE 2: The number range of the wounded after the earthquake.

\begin{tabular}{lc}
\hline $\begin{array}{l}\text { Time from earthquake } \\
\text { occurrence (days) }\end{array}$ & $\begin{array}{c}\text { The number range of } \\
\text { the wounded }\end{array}$ \\
\hline $0.5-1$ & {$[2168,6700]$} \\
$1-1.5$ & {$[6700,11393]$} \\
$1.5-2$ & {$[11393,11460]$} \\
$2-2.5$ & {$[11460,11470]$} \\
\hline
\end{tabular}

error of the upper and lower bounds and the comprehensive average simulation relative error of the model can be calculated. Then, the accuracy grade of the prediction model can be judged by referring to the continuous interval sequence error test table shown in Table 1.

\section{Prediction and Results Analysis}

Taking the Lushan earthquake in Sichuan Province on April 20,2013 , as an example, we use the continuous interval grey discrete Verhulst model based on the kernels and measures (CGDVM-KM) to forecast the number of the wounded. The data in this paper come from the statistics of earthquake patients (with an interval of 0.5 days) released by China Seismological Bureau (http://www.cea.gov.cn). See more details in Table 2.

Step 1. The kernel sequence is as follows:

$$
X^{(0)}(\widetilde{\otimes})=(4434,9046.5,11426.5,11465) .
$$

Step 2. The measure sequence is as follows:

$$
L^{(0)}=(4532,4693,67,10) .
$$

The first aggregate generator operator (1-AGO) of the measure sequence is

$$
L^{(1)}=(4532,9225,9292,9302) \text {. }
$$

Step 3. The simulation of the kernel sequence can be obtained as

$$
\widehat{\widetilde{\otimes}}=(4434,9046.49,11425.96,11465.26) .
$$

The simulation of the measure sequence's first aggregate generator operator (1-AGO) is

$$
\widehat{l}^{(1)}=(4532,9225.32,9291.99,9301.46) .
$$

The simulation sequence of the measure sequence can be obtained as 
TABLE 3: Upper and lower bound errors.

\begin{tabular}{|c|c|c|c|c|c|}
\hline & Time & $\begin{array}{c}\text { Actual value } \\
X(k)\end{array}$ & $\begin{array}{c}\text { Prediction value } \\
\widehat{X}(k)\end{array}$ & $\begin{array}{c}\text { Residual } \\
\varepsilon(k)=X(k)-\widehat{X}(k)\end{array}$ & $\begin{array}{c}\text { Relative error (\%) } \\
\Delta_{k}=|\varepsilon(k) / X(k)|\end{array}$ \\
\hline \multirow{4}{*}{ Lower bound } & 1 & 2168 & 2168 & 0 & 0.00000 \\
\hline & 2 & 6700 & 6699.83 & 0.17 & 0.00254 \\
\hline & 3 & 11393 & 11392.625 & 0.375 & 0.00329 \\
\hline & 4 & 11460 & 11460.525 & -0.525 & 0.00458 \\
\hline \multicolumn{5}{|c|}{ Average of relative error $\bar{\Delta}(1)=\left(\Delta_{2}+\Delta_{3}+\Delta_{4}\right) / 3$} & 0.00347 \\
\hline \multirow{4}{*}{ Upper bound } & 1 & 6700 & 6700 & 0 & 0.00000 \\
\hline & 2 & 11393 & 11393.15 & -0.15 & 0.00132 \\
\hline & 3 & 11460 & 11459.295 & 0.705 & 0.00615 \\
\hline & 4 & 11470 & 11469.995 & 0.005 & 0.00004 \\
\hline \multicolumn{5}{|c|}{ Average of relative error $\bar{\Delta}(2)=\left(\Delta_{2}+\Delta_{3}+\Delta_{4}\right) / 3$} & 0.00250 \\
\hline \multicolumn{5}{|c|}{ Comprehensive average relative error $\bar{\Delta}(3)=(\bar{\Delta}(1)+\bar{\Delta}(2)) / 2$} & 0.00299 \\
\hline
\end{tabular}

TABLE 4: Simulation results.

\begin{tabular}{lcr}
\hline Time from earthquake occurrence (days) & Original value & Prediction value \\
\hline $0.5-1$ & {$[2168,6700]$} & {$[2168,6700]$} \\
$1-1.5$ & {$[6700,11393]$} & {$[6699.83,11393.15]$} \\
$1.5-2$ & {$[11393,11460]$} & {$[11392.625,11459.295]$} \\
$2-2.5$ & {$[11460,11470]$} & {$[11460.525,11469.995]$} \\
$2.5-3$ & {$[11470,11470]$} & {$[11465.100,11466.440]$} \\
$3-3.5$ & {$[11470,11470]$} & {$[11465.685,11465.875]$} \\
$3.5-4$ & {$[11470,11470]$} & {$[11465.765,11465.795]$} \\
$4-4.5$ & {$[11470,11470]$} & {$[11465.780,11465.780]$} \\
$4.5-5$ & {$[11470,11470]$} & {$[11465.780,11465.780]$} \\
\hline
\end{tabular}

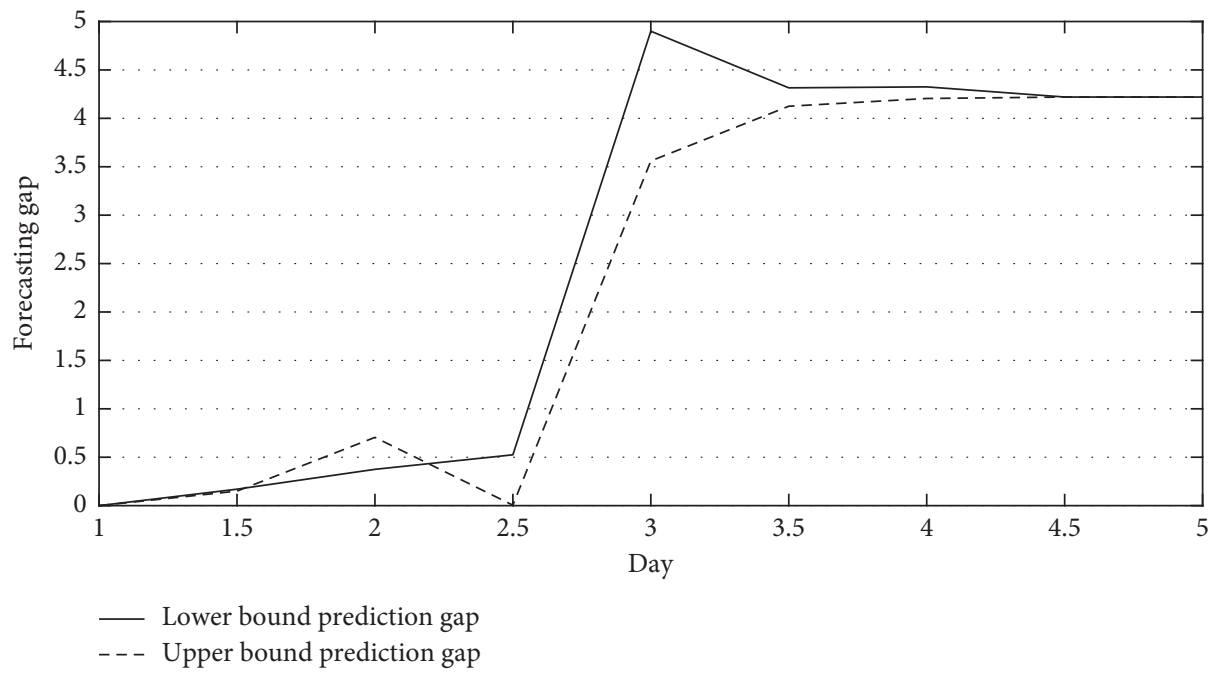

Figure 2: Prediction gap of the number of the wounded.

$$
\widehat{l}^{(0)}=(4532,4693.32,66.67,9.47) .
$$

Step 4. The simulated values of the lower bound sequence of the interval are as follows:

$$
\widehat{a}=(2168,6699.83,11392.625,11460.525) .
$$

The simulated values of the upper bound sequence of the interval are as follows:

$$
\widehat{b}=(6700,11393.15,11459.295,11469.995) .
$$

Step 5. From $\bar{\Delta}=(1 / n-1) \sum_{i=2}^{n} \Delta_{i}$, it can be concluded that the average relative error of the lower bound is $0.00347 \%$ and the average relative error of the upper bound is $0.00250 \%$. Thus, the average relative simulation error is $0.00299 \%$. See more details in Table 3.

From Table 3, we can see that the residual error of each data is below 1, which shows good fitting. The final 
TABLE 5: Comparison of prediction error between this model and other grey prediction models.

\begin{tabular}{lccccccc}
\hline Model & GVM-R & GDVM-R & CGDVM-GA & CGDVM-ID & CGGM-KM & CGVM-KM & CGDVM-KM \\
\hline Lower & $3.270 \%$ & $0.0218 \%$ & $13.579 \%$ & $0.0218 \%$ & $5.599 \%$ & $2.906 \%$ & $0.00347 \%$ \\
Upper & & $13.532 \%$ & $0.0153 \%$ & $4.866 \%$ & $1.841 \%$ & $0.00250 \%$ \\
Average & $3.270 \%$ & $0.0218 \%$ & $13.556 \%$ & $0.0185 \%$ & $5.233 \%$ & $2.374 \%$ & $0.00299 \%$ \\
\hline
\end{tabular}

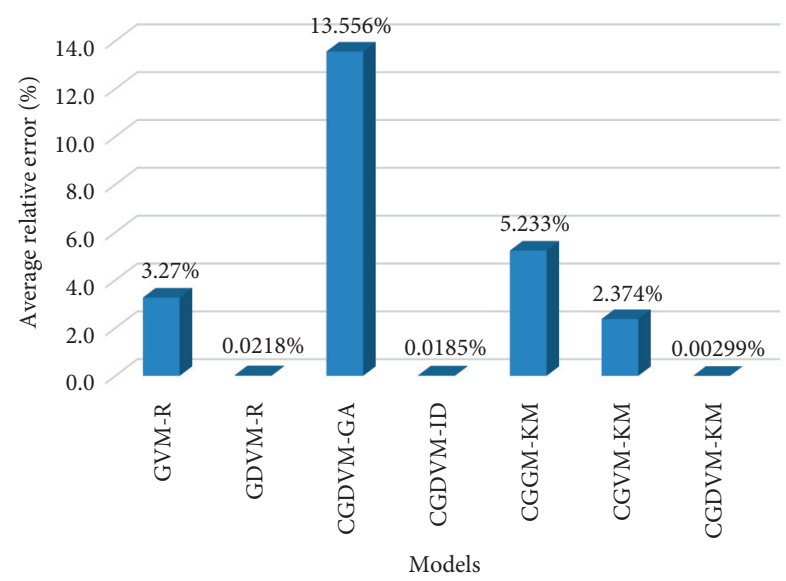

Figure 3: Prediction error of different models.

comprehensive average relative error is only $0.00299 \%$, which belongs to the first-stage prediction accuracy (see Table 1). It is shown that the model can accurately simulate the number of the wounded in the massive earthquake disaster. Table 4 shows the whole simulation results.

In reality, we know that, with further development of rescue operations, some patients with minor injuries will gradually recover and withdraw from treatment, and some patients who are seriously injured will be sent to large hospitals with better medical conditions in safe areas for further treatment after simple treatment. Therefore, the overall number of the wounded will decrease slightly, and then the total number of patients will gradually maintain a relatively stable level. From the results of interval prediction, we can see that, after a period of time, the interval forecast data are stable at a value and no longer change, which is consistent with the actual situation.

From Table 4 and Figure 2, we can see that the model constructed in this paper has a good effect on forecasting the number of the wounded in the massive earthquake disasters. To further illustrate the effectiveness of this model, the prediction results of the model constructed in this paper are compared with the prediction results of other models. The following six models are selected for comparison: (1) the real number grey Verhulst model (GVM-R), (2) the real number grey discrete Verhulst model (GDVM-R), (3) the continuous interval grey discrete Verhulst dynamic model based on grey attributes (CGDVM-GA), (4) the continuous interval grey discrete Verhulst dynamic model based on information decomposition (CGDVM-ID), (5) the continuous interval grey GM $(1,1)$ model based on kernels and measures (CGGM-KM), and (6) the continuous interval grey Verhulst model based on kernels and measures (CGVM-KM). Here, we divide the six models into three categories and compare them with the model constructed in this paper: the real number series models (i.e., model GVM-R and model GDVM-R), different interval whitening methods and the same prediction method models (i.e., model CGDVM-GA and model CGDVM-ID), and same interval whitening method and different prediction method models (i.e., model CGGM-KM and model CGVM-KM). The results are shown in Table 5 and Figure 3.

In the continuous interval grey prediction model, there are two key factors that affect the prediction accuracy: interval whitening method and grey prediction method. From Table 5, we can draw the following conclusions.

First, comparing with the real number grey prediction model, we find that the continuous interval grey prediction model has a higher prediction accuracy. This is because the whitening process of interval grey number can dig out more information from the data, and its prediction is more accurate. Second, under the same grey prediction method, we find that different whitening methods have a greater impact on the prediction results, and the whitening method based on kernels and measures has more advantages. This is because the whitening method is a way of interval data processing, and its purpose is to mine the information contained in interval data. The more the information is mined, the more accurate the prediction is. Finally, under the same whitening method, we find that different forecasting models have a great influence on the prediction results. The implied reason may be that the whitening method mines the interval data information, while the prediction method accurately and effectively fit the mined information, so as to predict the future trend. The grey discrete Verhulst model has more advantages than the grey GM $(1,1)$ model and the grey Verhulst model. In summary, the continuous interval grey discrete Verhulst model based on kernels and measures (CGDVM-KM) constructed in this paper has the highest simulation accuracy.

\section{Conclusions}

The continuous interval grey number has a more complex data structure than the real number and discontinuous interval grey number, so it is more difficult to build a model with higher prediction accuracy. In this paper, through the analysis of the regularity and characteristics of the statistical data of the number of the wounded after the massive earthquake with saturated "S-shape" and its continuous interval dynamic change trend, we consider the kernels and measures whitening method and grey discrete Verhulst prediction model and construct a continuous interval grey discrete Verhulst dynamic prediction model based on kernels and measures to predict the number of the wounded after the massive earthquake disasters. Compared with other real number and interval prediction models, the results show 
that the model constructed in this paper has higher prediction accuracy and better simulation effect and can more accurately and effectively simulate the number of the wounded after the massive earthquake disasters. Of course, the application of the prediction model is far more than the forecasting of the number of the wounded, and it has a good fitting for the data with saturated "S-shape" and continuous interval change trend.

At the same time, according to the research results of Feng et al. on the injury type and distribution data of the injured in Tangshan earthquake disaster and the medication data of the hospital for treating the injured in similar accidents, it can be seen that there is a linear correlation between the type and quantity of rescue drugs in earthquake disaster emergency rescue and the number of wounded people in an earthquake disaster [31]. Therefore, the continuous interval grey discrete Verhulst dynamic prediction model based on kernel sum measure can be used to predict the number of patients on the third day after the occurrence of Lushan earthquake disaster, and then, it is multiplied by the drug use coefficient of 40 kinds of conventional emergency relief drugs proposed in reference [31]. Finally, we can quickly and accurately calculate the type and quantity of emergency relief drugs on the third day after the Lushan earthquake disaster. Once the type and quantity of emergency relief drugs can be accurately and effectively, the headquarters and organizers of earthquake disaster relief will be able to timely and effectively allocate and supply the need for emergency relief medicines in earthquake-stricken areas, and the efficiency of earthquake disaster emergency relief can be improved.

Although we have constructed a model to effectively forecast the number of the wounded after massive earthquake disasters, the interval grey prediction model is a more complex model in the grey system theory, and there are many factors affecting its performance. Further optimization of the model is the direction of future research. In addition, the prediction of the number of the wounded is to better provide emergency relief materials, so how to more accurately correlate between the number of the wounded and materials needed is the focus of the next step.

\section{Data Availability}

The data used to support the findings of this study are included within the article.

\section{Conflicts of Interest}

The authors declare that there are no conflicts of interest.

\section{Acknowledgments}

This research was supported by the National Social Science Found of China (no. 10XGL013); Chongqing Social Sciences Planning Project (no. 2020TBWT09); Chongqing Municipal Education Commission Humanities and Social Sciences Research General Project (grant no. 18SKGH069); Scientific
Research Start-up Foundation of Chongqing Technology and Business University (no. 1855016); Chongqing Doctoral Program of Social and Scientific Planning (no. 2018BS79); and Open Research Fund of Chongqing Key Laboratory of Electronic Commerce and Supply Chain System (1456026).

\section{References}

[1] S. Chen and C. Wang, "Incorporating a bayesian network into two-stage stochastic programming for blood bank locationinventory problem in case of disaster," Discrete Dynamics in Nature and Society, vol. 2019, Article ID 7214907, 2019.

[2] J. Zhang, C. S. Chen, and B. Zeng, "Demand forecasting of emergency medicines after the massive earthquake-a grey discrete Verhulst model approach," Journal of Grey System, vol. 27, no. 3, pp. 234-248, 2015.

[3] E. Kayacan, B. Ulutas, and O. Kaynak, "Grey system theorybased models in time series prediction," Expert Systems with Applications, vol. 37, no. 2, pp. 1784-1789, 2010.

[4] X. Wu and J. Gu, "A modified exponential model for reported death toll during earthquakes," Earthquake Science, vol. 22, no. 2, pp. 159-164, 2009.

[5] H. X. Wang, J. X. Niu, and J. F. Wu, "ANN model for the estimation of life casualties in earthquake Engineering," Systems Engineering Procedia, vol. 1, no. 1, pp. 55-60, 2011.

[6] M. Gul and A. F. Guneri, "An artificial neural network-based earthquake casualty estimation model for Istanbul city," Natural Hazards, vol. 84, no. 3, pp. 1-16, 2016.

[7] X. Huang, M. J. Luo, and H. D. Jin, "Application of improved ELM algorithm in the prediction of earthquake casualties," Plos One, vol. 15, no. 6, pp. 1-13, 2020.

[8] X. Huang, J. Y. Song, and H. D. Jin, "The casualty prediction of earthquake disaster based on extreme learning machine method," Natural Hazards, vol. 102, pp. 873-886, 2020.

[9] E. Firuzi, K. Amini Hosseini, A. Ansari, Y. O. Izadkhah, M. Rashidabadi, and M. Hosseini, "An empirical model for fatality estimation of earthquakes in Iran," Natural Hazards, vol. 103, no. 1, pp. 231-250, 2020.

[10] F. S. Zhang, F. Liu, and W. B. Zhao, "Application of grey Verhulst model in middle and long-term load forecasting," Power System Technology, vol. 5, pp. 37-40, 2003.

[11] Z.-X. Wang, Y.-G. Dang, and S.-F. Liu, "Unbiased grey verhulst model and its application," Systems EngineeringTheory \& Practice, vol. 29, no. 10, pp. 138-144, 2009.

[12] L. Z. Cui, S. F. Liu, and Z. P. Li, "Grey discrete Verhulst model," Systems Engineering and Electronics, vol. 33, no. 3, pp. 590-593, 2011.

[13] M. Hashem-Nazari, A. Esfahanipour, and S. M. T. Fatemi Ghomi, "Non-equidistant "Basic Form"-focused grey verhulst models (Nbfgvms) for III-structured socio-economic forecasting problems," Journal of Business Economics and Management, vol. 18, no. 4, pp. 676-694, 2017.

[14] Z. X. Wang and Q. Li, "Modelling the nonlinear relationship between $\mathrm{CO}_{2}$ emissions and economic growth using a PSO algorithm-based grey Verhulst model," Journal of Cleaner Production, vol. 207, pp. 214-224, 2018.

[15] R. Rajesh, "Social and environmental risk management in resilient supply chains: a periodical study by the Grey-Verhulst model," International Journal of Production Research, vol. 57, no. 11-12, pp. 3748-3765, 2019.

[16] L. Wu and Z. Xu, "Analyzing the air quality of Beijing, Tianjin, and Shijiazhuang using grey Verhulst model," Air Quality Atmosphere and Health, vol. 12, no. 8, pp. 1-8, 2019. 
[17] B. Zeng, X. Ma, and M. Zhou, "A new-structure grey Verhulst model for China's tight gas production forecasting," Applied Soft Computing, vol. 96, 2020.

[18] R. Tian, Q. Shao, and F. Wu, "Four-dimensional evaluation and forecasting of marine carrying capacity in China: empirical analysis based on the entropy method and grey Verhulst model," Marine Pollution Bulletin, vol. 160, 2020.

[19] Y. F. Zhao, M. H. Shou, and Z. X. Wang, "Prediction of the number of patients infected with COVID-19 based on rolling grey Verhulst models," International Journal of Environmental Research and Public Health, vol. 17, no. 12, pp. 1-20, 2020.

[20] E. Liu, Q. Wang, X. Ge, and W. Zhou, "Dynamic discrete GM $(1,1)$ model and its application in the prediction of urbanization conflict events," Discrete Dynamics in Nature and Society, vol. 2016, Article ID 3861825, 2016.

[21] W. Zhou and D. Zhang, "An improved metabolism grey model for predicting small samples with a singular datum and its application to sulfur dioxide emissions in China," Discrete Dynamics in Nature and Society, vol. 2016, Article ID 1045057 , 2016.

[22] B. Zeng, S. Liu, and N. Xie, "Prediction model of interval grey number based on DGM $(1,1)$," Journal of Systems Engineering and Electronics, vol. 21, no. 4, pp. 598-603, 2010.

[23] B. Zeng and S. F. Liu, "Prediction model of interval grey number based on its geometrical characteristics," Journal of Systems Engineering, vol. 26, no. 2, pp. 1654-1663, 2011.

[24] M. Zhang, H. Wu, Z. Qiu, Y. Zhang, and B. Li, "Demand prediction of emergency supplies under fuzzy and missing partial data," Discrete Dynamics in Nature and Society, vol. 2019, Article ID 6823921, 2019.

[25] C. Li, Y. Yang, and S. Liu, "A greyness reduction framework for prediction of grey heterogeneous data," Soft Computing, vol. 2, pp. 17913-17929, 2020.

[26] B. Zeng, G. Chen, and S.-f. Liu, "A novel interval grey prediction model considering uncertain information," Journal of the Franklin Institute, vol. 350, no. 10, pp. 3400-3416, 2013.

[27] B. Zeng, C. Li, G. Chen, and W. Zhang, "Verhulst model of interval grey number based on information decomposing and model combination," Journal of Applied Mathematics, vol. 2013, pp. 1-8, 2013.

[28] J. Ye, Y. Dang, and Y. Yang, "Forecasting the multifactorial interval grey number sequences using grey relational model and GM $(1, N)$ model based on effective information transformation," Soft Computing, vol. 24, no. 7, pp. 5255-5269, 2020.

[29] L. Dang and L. I. Lin, "Prediction model of interval grey number based on kernels and measures," Mathematics in Practice and Theory, vol. 44, no. 8, 2014.

[30] B. Zeng, S. F. Liu, and J. Li, "An error inspection method for interval grey number prediction model based on kernel and interval length," in Proceedings of the IEEE International Conference on Grey Systems and Intelligent Services, Nanjing, China, October 2011.

[31] H. Feng, J. Li, S. Chen, M. Liu, and X. Sun, "Demand analysis of drugs for earthquake injury treatment," Journal of Pharmaceutical Practice, vol. 21, no. 2, pp. 100-102, 2003. 